\title{
Analysis on the Realization Method of Community Home Care Service Model in Xinjiang Alar
}

\author{
Shuhuan $\mathrm{He}^{1, \mathrm{a}}$, Chuanhui Zhang ${ }^{2, \mathrm{~b}}$
}

1,2 College of Economics and Management, Tarim University, Alar, Xinjiang, 843300

${ }^{\mathrm{a}}$ email, ${ }^{\mathrm{b}}$ email

Keywords: Xinjiang Alar, Community Home Care, Service Model

\begin{abstract}
Alar, southern Xinjiang geographical hinterland areas, is an important strategic fulcrum of stability and development in South, especially under the influence "along the way" strategic context, the economy has been rapid development, the number of elderly population has increased dramatically, how to better address the pension issue has become the focus of attention of government and society. Alar, Xinjiang community home care service model is based on community service network as the basis, the family unit as the carrier service model, service model content primarily for the care of older people's daily lives, while focusing on the elderly health care. This article Realize Alar, Xinjiang community home care service mode of conduct.
\end{abstract}

\section{Introduction}

China has entered the aging society stage, the elderly population is very large, with the arrival of an aging, whether urban development or industrial development are faced with enormous challenges, resulting in cross-regional occupational mobility continues to accelerate, so the elderly pension issues facing new challenges. At this stage, the traditional family pension continues to weaken, community home care service has a large advantage in terms of pension, therefore, each region should establish community home care model, an effective solution to the problem of population aging.

\section{Overview of Community Home Care Model}

Community home care model by Western countries in recent years proposed, to deal with the aging of society is an effective solution. China has entered the aging since many adults not only faced with the task of raising children, but also face very heavy pension burden, due to the social development of the pension immature, so the pension problems of the elderly has become a major social problem in order to solve this problem, we must develop an effective system for the elderly pension. Community home care service model is based on the family as the carrier, relying on a network of community service life of the elderly nursing care and carry out, at the same time spiritual solace and healthcare, to continuously improve the quality of life of older persons, so that older people can spend their serene later life. Community home care service model is a service to the community in the form of day-care and home services performed, professional services approach adopted pension institutions, not only make full use of domestic resources and services, as well as take advantage of a variety of community resources, social resources and personal resources from economic support, solace and other aspects of life care services for older people, home and social services will be effectively combined [1]. Community home care services in the home is no longer confined to a small home, but extends to older persons living in the community family.

Community home care service model includes services of three aspects, namely economic support, life care and spiritual solace. (1) China has entered the aging society since the economic pressures child support for the elderly is very large, community home care services in the economic support mainly refers to the different pension subject society, families, individuals and governments to provide each pension service model behaviors resources. Mainly, the economic will supports to provide financial resources for the retirement of the elderly. Community home care model of economic resources, including the source of three aspects, is a variety of community endowment 
insurance fund provided by the Government, the Government's financial allocation. The second is the mobilization of various financial community, the community raised a variety of social welfare pension including financial contributions, individual donations and social charitable donations, which is mainly used for the required pension contributions to the construction of various facilities, including a fitness facility, medical facilities and living facilities, many communities have established the elderly fitness facilities, facilities for the elderly in this area is generally in the name of the community receive the various contributions in society. In addition, the family of the elderly to provide financial resources, mainly for shelter, medical supplies and seriously ill elderly, children, housing for the elderly and household goods as well as financial and other elderly hospitalized provided. Overall, the economic support of the two main sources, namely to provide the community and families. (2) solace: Follow the elderly old age, not only to pay attention to the material life of the elderly, should also be concerned about the spiritual life of the elderly. The elderly after retirement, often faced with loneliness and isolation and other mental problems, and community home care service model should also be taken in various ways elderly rich spiritual life. (3) Life Care: Life Care Community Services for the Aged is a very important content, is also a basic services, life care including daily care and health care [2]. For the elderly, we have a healthy body is an important guarantee to spend a happy old age, so that health care services should be given higher priority.

\section{Feasibility of Alar Community Home Care Service Model}

Community home care model is in the aging society of social background, very consistent with China's national conditions. First, Alar government community home care service model formulated relevant policies, laying a solid foundation for the establishment of community home care service model, there is provided a system environment. Secondly, in recent years, Xinjiang's economy continues to develop, as the central region of Xinjiang Alar in southern Xinjiang, both in the industrial development or economic development have certain advantages, features and services are constantly improving the community, for the community home establish pension mode provided the conditions. In recent years, community building Alar, Xinjiang has the continuous development and advance. Many community service projects are constantly improving. Community service project contains the contents of the various aspects of basic necessities of life, to meet the daily lives of the elderly. Moreover, each community has a center for senior citizens, with related activities like chess clubs, fitness equipment, reading, etc., for the establishment of community home-based care model to provide the conditions.

In addition, with the continuous development of the regional economy, the service sector is also growing, the quality is improving community workers, community building itself contains issues cultural, economic and environmental aspects, it is a very Quantities large, and there is a certain degree of difficulty of the project. In the process of building the community we must rely on high-quality personnel. Currently, Alar government has recognized the importance of professionals in the community building. Finally, community home care service model simply extends the elderly home, community web-based, family support and social networks will not split the elderly, the elderly and therefore easier to accept. Using community home care service model to solve the pension problems of the elderly, the elderly do not need to leave their homes, the social environment has not changed, and the elderly can still contact their friends and relatives, so mentally not easy to feel lonely. The combination of these aspects, community home care model described in Alar, Xinjiang is feasible [3].

\section{Realization Method of Alar Community Home Care Service Model}

Community home care service model, the government plays an important role in order to achieve better community home care service model, we should give full play to the leading role of government functions and to provide policy and financial support for community home-based care. Therefore, the Government should continue to improve policies and foul on community home care 
service model, so as to promote the realization of community care model. Release opinion of Services for the Aged work, noting that community home care service model occupies a very important position in retirement services, We can promote the development of community home care model. In response to this has been introduced to the policy, the government should strengthen supervision, the policy will be carried out, in the actual implementation process continue to identify problems, look for shortcomings policy, and for lack of a place to be revised to make it continuous improvement [4].

Moreover, the government should strengthen the management of social welfare institutions, social welfare agencies, we can give some preferential policies, so as to give full play to the role of social welfare institutions in the community home-based care of. In addition, the government should establish a special community home care special fund to promote the development and implementation of community home care model, relying solely on policy support is not enough, they should also be given some help in the capital, the most important and direct form It is to establish a special fund. In addition to government funding and policy support, but also should give full play its advocacy role in strengthening the pension awareness publicity. Stage of China's social structure change dramatically, resulting in family pension function has also been weakened, children do not support the elderly in the community news often appears. In this case, the government should also strengthen the pension awareness publicity, and guide people to care for parents and respect for elders, in order to achieve community home-based care.

Community home care service model, the whole community is the project carrier, in order to better achieve the community home care service model, the community should give full play to their role in providing support and management support services for the community home care model. First, the community should build on existing community management system of community home care system, you can create community home care center management, develop appropriate Alar Development Community home-based care program based on government policies, but also should be developed for each species management practices and work systems, enabling communities to the correct implementation of the system and the policy community home care. In order to build a better service system, you can also set up a number of suggestion boxes, and the elderly residents themselves can be thought to establish and implement community home care model to provide comments or suggestions. In addition, communities can also set up community home care service stations, mainly the elderly in retirement needs to understand, is responsible for issuing various pension goods, services and pension services responsibilities of supervision and management, so as to continuously improve the quality of service of the pension agency.

We should also continue to improve existing community resources endowment, establish and improve the community home care service network. People's living standards continue to improve, demand is also rising, if a single pension service model, pension service quality will not improve. In response, the community should continue to improve existing community resources endowment, establish a more perfect community home care service network, you can create a multi-level network of community home care model, such as neighborhood support of community service, pure welfare community service, one community service and other service models, seniors can choose different types of service models according to their preferences and needs, so as to continuously improve the quality of service [5].

Pension demand different types of older age groups is not the same as in the establishment of community home care service model, but also to the needs of the elderly should be oriented to provide specialized services for the elderly. For example, can build community center for senior citizens, such as chess clubs, fitness room, painting room, a library, and chat station, so that younger seniors to actively participate in various activities Senior Center to address the elderly and lonely lone psychotic condition. Healthier of the body of the elderly can also actively encourage older people to participate in community volunteer activities center. For the oldest old, you can enrich the spiritual life by reading and talking with other means. Should also searched for spiritual solace mental health of the elderly, the elderly and therefore the community should also establish psychological assistance, lectures and actively carry out psychological counseling, eliminate 
adverse psychological factors in the elderly.

For the daily care of the elderly mainly in two ways, namely daycare services and home care, nursing home services to the elderly is entrusted to a number of services, the elderly will be pinned to the daycare center, you can choose different levels according to the actual economic situation the daycare center and service, daycare services provided itself into free services and low reimbursement services and paid services, different economic families can choose different types. Daycare service is very common in Western countries, older persons entrusted to daycare, nursing home can provide meals and simple care activities for the elderly. On-site service is also called home housekeeping, mainly through part-time nurse and asked people to volunteer and other forms of on-site service, on-site service personnel main task is to help the elderly care day life and do some simple daily chores [6]. Either way services should be based on the actual needs of the elderly as a guide, to provide specialized services.

\section{Conclusion}

The establishment of community home-based care services in Xinjiang Alar are available, in order to achieve community home care service model, first of all should play a leading role in the government, strengthening the pension awareness advocacy, policy support and financial support for community home care service model, followed by further should play to the community, we will establish service support system, continuous improvement of existing community resources endowment, establish and improve the community home care service network, and finally, it should be oriented to provide specialized services for the elderly according to demand.

\section{Acknowledgements}

New Century Talents Support Program (NCET-13-0925);

the Xinjiang Production and Construction Corps Excellence Project.

\section{References}

[1] Chang Yuanyuan. Path to build community home care model of Population Aging [J]. Journal of Hebei Software Institute, 2016, 13 (1): 10-13.

[2] Lin Yuyang, Zhong Manli. China path of feasibility pension model-Taking community mutual Perspective [J]. Agricultural Outlook, 2015, 36 (10): 89-90.

[3] Di Qingfei, Liu Jinhua. Social governance Horizon Changshou District of Chongqing community home care service model innovation to achieve the path [J]. Education Guide: Electronic Edition, 2016, 17 (4): 134-134.

[4] Li Lingzhi, Zhang Jiankun, Shi Dehua. Social organizations to participate in community home-based care and services model Construction [J]. Modern Urban Research, 2014, 32 (09): 1-7.

[5] Wang Wen. relying on low-income housing settlements to achieve re-employment of the elderly home care service model Construction [J]. Housing industry, 2015, 24 (6): 116-117.

[6] He Yu. Investigation and Feasibility demand "Pratt \& Whitney" community home-based care services and the Route of Realization - Shanghai Xuhui District in [D]. Fudan University, 2013. 